\title{
Agresividad y estado emocional en estudiantes de Instituciones Educativas de Lima Metropolitana
}

\author{
Aggressiveness and emotional state in students of Institutions Educational \\ Services of Metropolitan Lima
}

\author{
Marlon Manuel Omar Prado Verdi ${ }^{1}$ \\ Centro Juvenil de Diagnóstico y Rehabilitación de Lima
}

Recibido: $26-03-18$

Aceptado: $23-07-18$

\begin{abstract}
Resumen
Investigación de tipo descriptiva comparativa correlacional que tuvo como objetivo determinar los niveles de la agresividad - estado emocional y establecer la relación y comparación de la agresividad y el estado emocional en función del sexo y tipo de familia a la que pertenecen. Para tal efecto se aplicaron a una muestra de 343 escolares del 1 ro al 4to año de secundaria, de ambos sexos, de dos instituciones educativas de Lima Metropolitana, el Inventario de Hostilidad Agresividad de Buss-Durkee (1987) y la escala de auto percepción del estado emocional (Vila Doris - Díaz Luis, 2007). Los datos se procesaron en el programa estadístico SPSS versión 22.0 en español y se llegaron a las siguientes conclusiones: En agresividad el $18.1 \%$ de la muestra estudiada presentan niveles de agresividad que va desde el nivel alto al nivel excesivo. En estado emocional el $28.5 \%$ de la muestra presentan niveles moderados a niveles altos. Se encuentra una relación moderada positiva muy significativa entre agresividad y el estado emocional. En estado emocional no existen diferencias en función del sexo.

Palabras clave: Agresividad; estado emocional; instituciones educativas; estudiantes; Lima Metropolitana.
\end{abstract}

\begin{abstract}
Correlative descriptive comparative type research that aimed to determine the levels of aggressiveness - emotional state and establish the relationship and comparison of aggressiveness and emotional state depending on the sex and type of family to which they belong. For this purpose, a sample of 343 school children from the 1st to 4th year of secondary school, of both sexes, from two educational institutions in Metropolitan Lima, the Buss-Durkee Hostility Aggression Inventory (1987) and the self-perception scale of the emotional state (Vila Doris - Díaz Luis, 2007). The data were processed in the statistical program SPSS version 22.0 in Spanish and the following conclusions were reached: In aggressiveness, $18.1 \%$ of the studied sample presented levels of aggressiveness ranging from the high level to the excessive level. In emotional state, $28.5 \%$ of the sample presented moderate levels at high levels.
\end{abstract}

1 Psicólogo del Centro Juvenil de Diagnóstico y Rehabilitación de Lima. Email: mp_verdi@hotmail.com

(C) Los autores. Este artículo es publicado por la Revista de Investigación en Psicología de la Facultad de Psicología, Universidad Nacional Mayor de San Marcos. Este es un artículo de acceso abierto, distribuido bajo los términos de la licencia Creative Commons Atribucion - No Comercia_Compartir Igual 4.0 Internacional. (http://creativecommons.org/licenses/by-nc-sa/4.0/) que permite el uso no comercial, distribución y reproducción en cualquier medio, siempre que la obra original sea debidamente citada. 
A very significant positive moderate relationship between aggressiveness and emotional state is found. In emotional state there are no differences depending on the sex.

Keywords: Aggressiveness; emotional behavior; educational institutions; students; Metropolitan Lima.

Diversos autores señalan que la agresividad se puede manifestar en diferentes maneras de violencia, dependiendo del contexto, puede ser manifestar en el área social, familiar, escolar, etc. En el área social se puede reconocer el vandalismo, el pandillaje, peleas callejeras, las barras bravas. En el área familiar, se distingue la violencia entre los padres, de padres hacia hijos, entre los hijos o de los hijos hacia los padres. En el área escolar podemos reconocer la agresividad profesor hacia el alumno, el alumno hacia el profesor, alumno hacia alumno y el tan mencionado bullying.

Para Parra (1998) cuando un estudiante ingresa a un grupo de pares se dan dos corrientes: el interés de dominación y el de adhesión. La conducta de agresión a través de pares se debería a la disgregación que sobrellevan cuando se incorporan al entorno social entre pares. Quedando relegado el entorno familiar y aparece el interés de aceptación y se ve influenciado por la moda y el entorno. Pero su interés y acción han de encontrarse con los de otros, provocando una pugna que se solucionara por medio del temperamento e historia personal. Es así, que decidirá por esforzarse para imponerse sobre el otro, se doblegará a él, o se aislará.

Algunas investigaciones han evidenciado que los adolescentes agresores alcanzan niveles de media en popularidad. Estos estudiantes encuentran afinidad en un reducido grupo de amigos que son cómplices y se asemejan con ellos. No obstante, la fama disminuye en los grados superiores. Peor los alumnos violentos no llegan a un nivel bajo de popularidad que singulariza a las víctimas (Olweus, 2004).

Al respecto Garaigordobil (2005) refiere que en la juventud la agresividad se relaciona a comportamientos tales como destruir bienes de la comunidad u objetos que pertenecen a otras personas, faltas constantes al colegio, consumo excesivo de alcohol y/o sustancias, etc.

Asimismo, en cuanto al acto de agresividad, acoso, violencia, etc., hay sujetos agresores y sujetos agredidos; en ambos casos, no se puede negar que el estado emocional está siendo afectado generando problemas de ansiedad, depresión, conductas de evitación, retraimiento, aislamiento, etc. Al respecto Cerezo (2001) al analizar la variable Neuroticismo no mostró diferencias significativas entre los grupos de sujetos agresores y los sujetos víctimas (el estado emocional es similar en muchos aspectos al rasgo de neuroticismo propuesto por Eysenck H en 1983). Algunas de las características de los adolescentes con conducta agresiva son el 
bajo rendimiento académico, elevadas cogniciones prejuiciosas, baja capacidad de empatía y alta impulsividad. De hecho, rasgos tales como impulsividad, hostilidad e inestabilidad emocional, se relacionan con la conducta delictiva (Garaigordobil, 2005).

Buss y Perry (1992) consideran que las reacciones agresivas tienen dos componentes: la expresión de impulsos aversivos y el ambiente interpersonal; dando la siguiente definición a la agresión: "reacción que descarga estímulos nocivos sobre otros organismos". La agresividad se puede percibir a nivel conductual, emocional y cognitivo. A nivel conductual tenemos la agresión física y la agresión verbal. Y a nivel emocional encontramos a la ira y a nivel cognitivo la hostilidad.

Al respecto (López, Sánchez, Pérez y Fernández, 2008) refieren que "la agresividad es el resultado de un engorroso aprendizaje por asociación en donde influyen procesos biológicos, de aprendizaje, conductuales, cognitivos y emocionales".

Por otro lado al hablar del estado emocional (Chóliz, 2005) menciona que cualquier proceso cognitivo implicaría una respuesta emocional de mayor o menor intensidad y magnitud. Considerando así que en toda reacción emocional estará presente procesos cognitivos.

La idea de inestabilidad emocional es parecida en muchos aspectos al rasgo de neuroticismo expuesto por Eysenck $\mathrm{H}$. El Neuroticismo se refiere a emociones negativas y alude problemas de personalidad e implicaría que la inestabilidad es el resultado de la reacción emocional sin importar que emoción (ansioso, deprimido, sentimiento de culpa, poca autoestima, tensión) (Eysenck, 1983).

En resumidas cuentas, el estado emocional se refiere a la respuesta de los procesos cognitivos que el individuo muestra al enfrentar estímulos que le generan agrado o desagrado. El estado emocional siempre se presenta conductualmente con cambios en los procesos neurovegetativos e implica dificultad en dar una respuesta adecuada a la intensidad y magnitud de un estímulo. (Vila y Díaz, 2007).

\section{Objetivos generales}

- Determinar los niveles de la agresividad y el estado emocional de los estudiantes de dos instituciones educativas de Lima Metropolitana.

- Establecer la relación y comparación de la agresividad y el estado emocional de los estudiantes de dos instituciones educativas de Lima Metropolitana en función del sexo a la que pertenecen. 


\section{Objetivos específicos}

1. Describir los niveles de agresividad de los estudiantes de instituciones educativas de Lima Metropolitana.

2. Describir los niveles del estado emocional de los estudiantes de instituciones educativas de Lima Metropolitana.

3. Relacionar la agresividad con el estado emocional de los estudiantes de instituciones educativas de Lima Metropolitana.

4. Comparar los promedios de la agresividad de los estudiantes de instituciones educativas de Lima Metropolitana en función del sexo.

5. Comparar los promedios del estado emocional de los estudiantes de instituciones educativas de Lima Metropolitana en función del sexo.

\section{METODOLOGÍA}

\section{Tipo de investigación}

Es descriptiva porque identifica y describe las características de las variables de estudio.

\section{Diseño de investigación}

Es de tipo comparativo - correlacional.

Ex post facto, dado que los datos a obtenerse ya están presentes en los estudiantes encuestados (Kerlinger, 2004)

\section{Población y muestra}

La población a estudiarse estuvo conformada por 1800 escolares matriculados en 1ro (225 alumnos), 2do (225 alumnos), 3er (225 alumnos) y 4to (225) año de secundaria de cada una de las 02 instituciones educativas nacionales (IE. República Federal Alemania y la IE Perú Valladolid) de Lima Metropolitana. Hombres y mujeres matriculados del primer a cuarto año de secundaria.

Las muestras de la presente investigación, fueron seleccionadas de manera no probabilística e intencionada y estuvo constituida por un grupo de 316 escolares que fueron evaluados intencionalmente y estuvieron de acuerdo a participar en forma voluntaria. La muestra representa al $17.56 \%$ de la población total estudiada. Sin embargo, dado que la selección es de tipo no probabilística e intencionada se evaluó a 343 escolares, que corresponde al 19.05\% de la población total. Al describir la distribución de la muestra según el sexo el 50.7\% pertenecen al sexo masculino y el $49.3 \%$ pertenecen al sexo femenino. 


\section{Instrumentos}

\section{a. El Inventario de Hostilidad Agresividad de Buss-Durkee}

La prueba de Hostilidad Agresividad de Buss-Durkee, fue adaptada en nuestro país por el Licenciado en Psicología Reyes Romero, Carlos; de la Universidad Ricardo Palma en 1987). Los reactivos del Inventario de Hostilidad Agresividad de BussDurkee de 75 reactivos divididos de la siguiente manera. Violencia. Hostilidad indirecta. Irritabilidad. Negativismo. Resentimiento. Recelos. Hostilidad verbal. Culpabilidad. La confiabilidad del inventario de agresividad, se halló a través del alpha de Cronbach, dando como resultado general 0.45 . Respecto a la validez se utilizó el coeficiente de Pearson, y en la correlación se utilizó el corrector de McNemar, obteniendo en la escala de irritabilidad 0.30; verbal 0.32; indirecta 0.31 ; física 0.27 ; resentimiento 0.32 ; sospecha 0.29 .

\section{Validez y confiablidad}

Respecto a la validez del constructo para la presente investigación, los índices de correlación de Pearson están en un rango de 0.52 a 0.77 , estos valores afirman que presenta validez en sus puntuaciones. Referente a la confiabilidad, el coeficientes Alpha son elevados en todas los ítems de la escala de agresividad, porque superan los valores de 0.60 , aceptado como adecuado para los instrumentos psicológicos. Así como la escala en su totalidad presenta un valor Alpha $=\mathbf{0 . 7 1 3 8}$ que es fuerte o elevado (véase anexo 3).

\section{b. Escala de auto percepción del estado emocional (Vila y Díaz, 2007)}

La escala está conformada por 35 ítems. Es una escala de medición directa con respuestas randomizadas con 04 alternativas de respuesta. $\mathrm{Su}$ aplicación puede ser individual o grupal. El tiempo de aplicación es de 15 a 20 minutos. La escala obtuvo una validez de contenido con el coeficiente Aiken de 0.84. Así mismo se aplicó el Coeficiente de Cronbach para hallar la confiabilidad del instrumento Auto percepción del Estado Emocional correspondiente dando un coeficiente de $0.83\left(\mathrm{~N}^{\circ}\right.$ casos 300$)$ lo que señala una muy buena confiabilidad del instrumento.

\section{Validez y confiablidad}

Respecto a la validez del instrumento todos los Coeficientes de Correlación de Pearson ( $r)$ son fuertes y muy significativos $(\mathrm{p}<0.01)$. Dado que los índices de correlación de Pearson están en un rango de 0.739 a 0.925 que son valores altos y muy altos (Ávila 1998). Asimismo la escala de estado emocional presenta referente a la confiablidad se presenta en sus puntuaciones; dado que el coeficientes Alpha son elevados en todas los ítems de la escala del estado emocional, ya que superan los valores de 0.70 (véase anexo 4) 


\section{RESULTADOS}

\section{Describir los niveles de agresividad}

En la tabla 1, se describe la distribución de la muestra por niveles de agresividad, reportándose que el $61.2 \%$ están catalogados en un nivel moderado de agresividad; que el $18.1 \%$ de la muestra están catalogados en un nivel de agresividad leve y que el $17.5 \%$ presenta un nivel alto de agresividad. El $0.6 \%$ están catalogados dentro del nivel excesivo de agresividad. Sólo un $2.6 \%$ de la muestra que reporta un nivel normal de agresividad.

Tabla 1

Distribución por niveles de agresividad

\begin{tabular}{lcc}
\hline Niveles escala general & Frecuencias & Porcentajes \\
\hline Permitido/Normal & 9 & 2.6 \\
Leve & 62 & 18.1 \\
Moderado & 210 & 61.2 \\
Alto & 60 & 17.5 \\
Excesivo & 2 & 0.6 \\
Total & 343 & 100 \\
\hline
\end{tabular}

\section{Describir los niveles del estado emocional.}

En la tabla 2, se observa que el 58.3\% están catalogados en un nivel leve del estado emocional; que el $26.5 \%$ de la muestra están catalogados en un nivel moderado y tan solo el $2 \%$ presenta un nivel alto. Sólo el $13.1 \%$ están catalogados dentro del nivel normal.

Tabla 2

Distribución de la muestra por niveles de conducta emocional

\begin{tabular}{lcc}
\hline \multicolumn{1}{c}{ Niveles conducta emocional } & Frecuencias & Porcentajes \\
\hline Permitido/Normal & 45 & 13.1 \\
Leve & 200 & 58.3 \\
Moderado & 91 & 26.5 \\
Alto & 7 & 2 \\
Total & 343 & 100 \\
\hline
\end{tabular}

\section{Establecimiento de correlaciones.}

\section{A través del estadístico paramétrico Producto momento de Pearson}

En la tabla 3, no hay relación entre las variables de estudio, donde se concluye que existe una correlación moderada positiva estadísticamente muy significativa conducta emocional y agresividad $\left(\mathrm{r}=.585^{* *}\right)$. 
Tabla 3

Correlación entre agresividad y conducta emocional (escalas generales)

\begin{tabular}{lcc}
\hline & \multicolumn{2}{c}{ Conducta emocional } \\
\cline { 2 - 3 } & $\mathbf{R}$ & $\mathbf{P}$ \\
\hline Agresividad & $0.585^{* *}$ & .001 \\
\hline
\end{tabular}

Decisión estadística: Se rechaza la Ho (no existe relación entre agresividad y conducta emocional)

En la Tabla 4, se concluye que existe una correlación baja positiva estadísticamente muy significativa entre conducta emocional las dimensiones de hostilidad indirecta, Violencia, Irritabilidad negativismo y recelo (sus valores $\mathrm{r}$ oscilan entre $0.333^{* *}$ a $0.390^{* *}$ ). Existe correlación positiva moderada altamente significativa con las dimensiones resentimiento, hostilidad verbal y culpabilidad.

\begin{tabular}{lcc}
\begin{tabular}{l} 
Tabla 4 \\
$\begin{array}{l}\text { Correlación dimensiones de agresividad y conducta emocional (escala } \\
\text { general) }\end{array}$ \\
\hline Agresividad
\end{tabular} & \multicolumn{2}{c}{ Conducta emocional } \\
\cline { 2 - 3 } Violencia & $\mathbf{R}$ & $\mathbf{P}$ \\
Hostilidad indirecta & $.389^{* *}$ & .001 \\
Irritabilidad & $.333^{* *}$ & .001 \\
Negativismo & $.360^{* *}$ & .001 \\
Resentimiento & $.390^{* *}$ & .001 \\
Recelo & $.524^{* *}$ & .001 \\
Hostilidad verbal & $.375^{* *}$ & .001 \\
Culpabilidad & $.598^{* *}$ & .001 \\
\hline
\end{tabular}

** La correlación es altamente significativa $(\mathrm{p}<.01)$

\section{Establecimiento de comparaciones de la Agresividad.}

\section{Según sexo}

En la siguiente Tabla 5, se establece la comparación de la agresividad en función del sexo, reportándose que no hay diferencias entre los promedios de las variables de estudio en función del sexo.

\section{Tabla 5}

Establecimiento de comparaciones en agresividad en función del sexo.

\begin{tabular}{lcccccc}
\hline Sexo & N & Media & DS & T & Gl & P \\
\hline Varones & 174 & 37.23 & 7.190 & -0.657 & 341 & .445 \\
Mujeres & 169 & 37.18 & 6.247 & & & \\
\hline
\end{tabular}




\section{Establecimiento de comparaciones del Estado emocional}

\section{Según sexo}

En la Tabla 6, se establece las diferencias de la conducta emocional en función del sexo, se acepta que no hay diferencias entre los promedios de conducta emocional en función del sexo.

Tabla 6

Establecimiento de la diferencias de promedios de la conducta emocional en función del sexo.

\begin{tabular}{lcccccc}
\hline Sexo & $\mathbf{N}$ & Media & DS & T & Gl & P \\
\hline Varones & 174 & 34.20 & 12.61 & -1.48 & 341 & .148 \\
Mujeres & 169 & 36.21 & 12.59 & & & \\
\hline
\end{tabular}

\section{ANÁLISIS Y DISCUSIÓN DE LOS RESULTADOS}

Al describir los niveles de agresividad, se encontró un considerable porcentaje $97.4 \%$ de alumnos presentan niveles de agresividad que va desde lo leve al nivel alto lo que implica según la teoría mimética, que las relaciones humanas son conflictivas y violentas; la tranquilidad es momentánea. Asimismo, un 18.1\% presentó niveles de agresividad que va desde un nivel alto al nivel excesivo al relacionarse con los otros, siendo alumnos de alto riesgo. Lo cual implica que debido a su alto nivel de agresividad, su estilo agresivo podría ser la puerta de entrada a comportamientos disóciales empezando con la deserción escolar, consumo de drogas legales e ilegales, pequeños hurtos, pertenecía a pandillas, etc. Garaigordobil (2005).

En cuanto a los niveles de la conducta emocional; el 28.5\% de la muestra presentan niveles de moderado a alta emocionalidad. Al respecto, Contini, Imach, Coronel y Mejail (2012) encontraron que el 19.8\% de adolescentes advirtieron problemas al establecer vínculos sociales, manifestaron sensación de vergüenza, timidez y tension (estado emocional). Asimismo, tan solo el 2\% presenta un nivel alto de estado emocional; estos resultados coinciden con los de Barrio, Moreno y López (2001) que al analizar la variable de inestabilidad emocional en púberes españoles estimaron que la tasa asciende al 2.07\% de la muestra.

Respecto a la relación entre agresividad y estado emocional, concluimos que existe una correlación moderada positiva. Como se corrobora en el estudio de Gutiérrez, Hernández, Sebastián y Suárez (2000) quienes encontraron que el neuroticismo obtuvo una correlación positiva con la conducta delictiva. Del mismo modo, Contini, et al. (2012) obtuvieron relaciones significativas positivas entre los factores agresividad y aislamiento. Implicaría que cuando el adolescente manifiesta conductas agresivas en sus relaciones interpersonales, predominando sus intereses por sobre el de los demás, puede producir en su par comportamientos 
de rechazo; por ello, las nuevas oportunidades de vínculos se verán afectadas potenciando conductas emocionales inestables.

Al relacionar el estado emocional con las dimensiones de la agresividad, concluimos que existe una correlación positiva moderada altamente significativa con las dimensiones resentimiento, hostilidad verbal y culpabilidad (los índices $r$ oscilan entre 0.525 a 0.598 ) destacándose como los principales factores predictores para que se manifieste la inestabilidad emocional. Al respecto, Mestre, Samper, Frias (2004) al analizar el comportamiento agresivo y los procesos emocionales en la adolescencia; indicaron que el comportamiento agresivo alcanza una mayor correlación con los procesos emocionales.

En cuanto al comparar los promedios de la agresividad en función al sexo. Se encontró que no hay diferencias entre los promedios de agresividad en función del sexo. Estos resultados coinciden con los resultados de Araujo (2013) que al establecer comparaciones de la agresividad en función del sexo, no halló diferencias entre géneros en dominancia, ajuste social, agresividad, integridad y deseabilidad social. Sin embargo Matalinares et al. (2010) dijeron que la agresividad de los estudiantes se muestra diferente en función del sexo al hallarse diferencias significativas en la subescala de agresividad física entre varones y mujeres. En efecto en las encuestas los hombres admiten ser más agresivos que las mujeres. En los experimentos de laboratorio, los hombres muestran, una mayor agresión física. Al respecto, Bettencourt y Miller (1996) refiere que las diferencias de género dependen del contexto. Cuando hay provocación, la diferencia entre género se reduce y dentro de las formas de agresión menos ofensivas (atacar verbalmente, dar una bofetada, tirar algo) las mujeres no son menos agresivas que los hombres.

Al comparar los promedios del estado emocional en función al sexo. Se acepta que no hay diferencias entre los promedios de conducta emocional en función del sexo. Al respecto, Contini et al. (2012), al establecer comparaciones según el género no se registraron diferencias significativas en función de la inestabilidad emocional (aislamiento y retraimiento/ansiedad)

\section{CONCLUSIONES}

1. El $18.1 \%$ presentan niveles de agresividad que va desde alto al nivel excesivo al relacionarse con los otros. Lo cual implica que debido a su alto nivel de agresividad, su estilo agresivo podría ser la puerta de entrada a comportamientos disóciales; empezando con la deserción escolar, consumo de drogas legales e ilegales, pequeños hurtos, pertenecía a pandillas, etc.

2. En estado emocional el $28.5 \%$ de la muestra presentan niveles de moderado estado emocional a alta emocionalidad. Lo cual supondría 
que los sujetos advirtieron problemas al establecer vínculos sociales, manifestando vergüenza, timidez, tensión, etc.

3. Se encuentra una relación moderada positiva muy significativa entre agresividad y el estado emocional. Destacándose la agresividad como un factor predictor para que se manifieste la inestabilidad emocional.

4. No existen diferencias significativas de agresividad en función del sexo. Implicaría que cuando hay provocación, la diferencia entre género se reduce y dentro de las formas de agresión menos ofensivas (atacar verbalmente, dar una bofetada, tirar algo) las mujeres no son menos agresivas que los hombres.

5. En estado emocional no existen diferencias en función del sexo. Dicho resultado son corroborados por diversas investigaciones.

\section{ANEXO}

\section{Anexo 1:}

\section{ESCALA DE AG MANIFIESTA}

\section{INSTRUCCIONES}

En las siguientes páginas se le presenta una serie de frases sobre el modo como Ud., se comporta como Ud., se y siente. Después de leer cada frase, debe decidir con un "CIERTO" o con un "FALSO" a aquella que represente su modo de actuar o sentir usualmente.

Trate de responder rápidamente y no emplee mucho tiempo en cada frase; queremos su primera reacción, no un proceso de pensamiento prolongado.

\section{ASEGURESE DE NO OMITIR ALGUNA FRASE.}

Ahora trabaje rápidamente y recuerde de contestar todas las frases. NO HAY CONTESTACIONES "CORRECTAS" O "INCORRECTAS", sino simplemente una medida de la forma como Ud. Se comporta.

Para responder, ponga una equis $(\mathrm{X})$ debajo de la columna "C" (cierto) o "F" (Falso).

1 Pierdo la paciencia fácilmente, pero la recobro fácilmente.

2 Cuando desapruebo la conducta de mis amigos (as) se los hago saber.

3 A veces hablo mal de las personas que no me agradan.

4 De vez en cuando no puedo controlar mi necesidad de golpear a otros.

5 Siento que no consigo lo que merezco.

6 Sé de personas que hablan de mí a mis espaldas.

7 Siempre soy paciente con los demás.

8 A menudo me encuentro en desacuerdo con los demás.

9 Nunca me molesto tanto como para tirar las cosas.

10 No tengo ninguna buena razón para golpear a los otros. 
11 Otra gente parece que lo obtiene todo.

12 Me mantengo en guardia con gente que de alguna manera es más amigable de lo que esperaba.

13 Soy más irritable de lo que la gente cree.

14 No puedo evitar entrar en discusiones cuando la gente no está de acuerdo conmigo.

15 Cuando me molesto, a veces tiro las puertas.

$16 \mathrm{Si}$ alguien me golpea primero, le respondo de igual manera y de inmediato.

17 Cuando recuerdo mi pasado y todo lo que me ha sucedido, no puedo evitar sentirme resentido (a).

18 Creo que le desagrado a mucha gente.

19 Me "hierve" la sangre cada vez que la gente se burla de mí.

20 Yo exijo que la gente respete mis derechos.

21 Nunca hago bromas pesadas.

22 Quien sea que insulte a mi familia o a mí, está buscando pelea.

23 Casi todas las semanas encuentro a alguien que me desagrada.

24 Hay mucha gente que me tiene envidia.

25 Si alguien no me trata bien, no permito que eso me moleste.

26 Aún cuando estoy enfurecido no hablo lisuras.

27 A veces hago mal las cosas cuando estoy enojado (a).

28 Quien continuamente me molesta, está buscando un puñete en la nariz.

29 Aunque no lo demuestre, a veces siento envidia.

30 A veces tengo la sospecha de que se ríen de mí.

31 A veces me molesta la sola presencia de la gente.

32 Si alguien me molesta estoy dispuesto (a) a decirle lo que pienso.

33 A veces dejo de hacer las cosas cuando no consigo lo que quiero.

34 Pocas veces contesto, aunque me golpeen primero.

35 No sé de alguien a quien odie completamente.

36 Mi lema es "nunca confiar en extraños".

37 A menudo me siento como "pólvora a punto de estallar".

38 Cuando la gente me grita, les grito también.

39 Desde $\operatorname{los} 10$ años no he tenido una rabieta.

40 Cuando verdaderamente pierdo la calma, soy capaz de cachetear a alguien.

41 Si permito que los demás me vean como soy, seré considerado difícil de llevar.

42 Comúnmente pienso, que razón oculta tendrán para hacer algo bueno por mí.

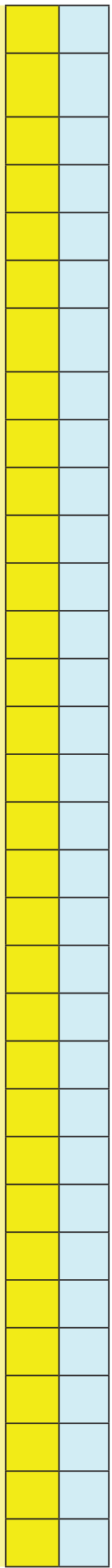


43 A veces me siento "acalorado" (a) y de mal genio.

44 Cuando me molesto digo cosas desagradables.

45 Recuerdo que estuve tan amargo que cogí lo primero que encontré a mano y lo rompí.

46 Peleo tanto como las demás personas.

47 A veces siento que la vida me ha tratado mal.

48 Solía pensar que la mayoría de la gente decía la verdad, pero ahora sé que estoy equivocado (a).

49 No puedo evitar ser rudo (a) con la gente que no me agrada.

50 No puedo poner a alguien en su lugar, aún si fuese necesario.

51 A veces demuestro mi enojo golpeando la mesa.

52 Si tengo que recurrir a la violencia física para defender mis derechos, lo hago.

53 Aunque no lo demuestre, me siento insatisfecho (a) conmigo mismo (a).

54 No tengo enemigos que realmente quieran hacerme daño.

55 No permito que muchas cosas sin importancia me irriten.

56 A menudo hago amenazas que no cumplo.

57 Cada vez que estoy molesto (a) dejo de hacer las tareas de mi casa.

58 Sé de personas que por molestarme, me han obligado a usar la violencia.

59 Hay personas a quienes les guardo mucho rencor.

60 Raramente siento que la gente trata de amargarme o insultarme.

61 Últimamente he estado algo malhumorado.

62 Cuando discuto tiendo a elevar la voz.

63 Me desquito una ofensa negándome a realizar las tareas.

64 Quienes me insultan sin motivo, encontrarán un buen golpe.

65 No puedo evitar ser tosco con quienes trato.

66 He tenido la impresión de que ciertas personas me han tratado de sacar provecho.

67 Me irrita rápidamente cuando no consigo lo que quiero.

68 Generalmente oculto la pobre opinión que tengo de los demás.

69 Cuando me molestan me desquito con las cosas de quienes me desagradan.

70 Quien se burla de mis amigos (as) se enfrentará a golpes conmigo.

71 Me duele pensar que mis padres no hicieron lo suficiente por mí.

72 A veces desconfío de las personas que tratan de hacerme un favor.

73 Tiendo a irritarme cuando soy criticado.

74 Prefiero ceder en algún punto de vista antes de discutir.

75 Con mis amigos acostumbro a burlarme de quienes no me agradan.

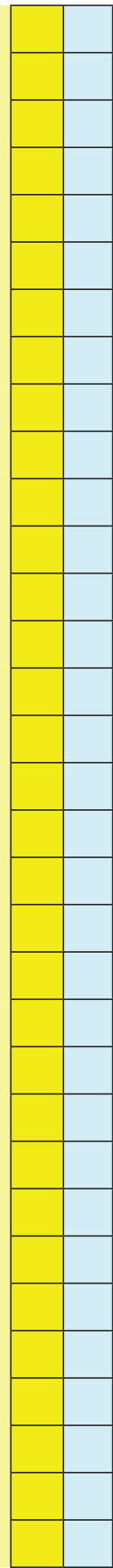




\section{Anexo 2:}

\section{Escala Autopercepción de la Conducta Emocional}

Instrucciones: Aquí tienes en esta escala un conjunto de 35 items, que describe tus comportamientos. Lo que tienes que hacer es marcar con una $\mathrm{x}$ en una de las columnas de tu derecha que te corresponde.

\section{Alternativas: S: SIEMPRE MV: MUCHAS VECES AV: A VECES N: NUNCA}

\begin{tabular}{|c|c|c|c|c|c|}
\hline $\mathbf{N}^{\mathbf{a}}$ & ÍTEMS ESCALA & $\mathbf{S}$ & MV & AV & $\mathbf{N}$ \\
\hline 1 & $\begin{array}{l}\text { Reconozco que he sido más agresivo de lo normal con mis compañeros del aula, pero } \\
\text { después me he arrepentido. }\end{array}$ & & & & \\
\hline 2 & Tengo pensamientos de que los demás son mejor que yo y eso me pone de mal humor. & & & & \\
\hline 3 & $\begin{array}{l}\text { El nivel económico en mi familia no cubre todo lo que a mí me gustaría tener y eso me } \\
\text { llena de ira por que no puedo tener lo que mis compañeros (a) tienen (juegos, propinas, etc) }\end{array}$ & & & & \\
\hline 4 & Me he sentido culpable por haber perjudicado involuntariamente a mis compañeros. & & & & \\
\hline 5 & Me suelo emocionar fácilmente. Creo que soy una persona sensible. & & & & \\
\hline 6 & Me gusta estar solo (a) y poder relajarme a escondidas de mis padres. & & & & \\
\hline 7 & Últimamente me siento solo y salgo menos con los amigos (as) de antes. & & & & \\
\hline 8 & Pienso que la vida es bastante aburrida. & & & & \\
\hline 9 & Para tomar decisiones necesito consultar a mis amigos(as). & & & & \\
\hline 10 & Pienso que dios es injusto por el tipo de vida que llevo. & & & & \\
\hline 11 & Soy tolerante cuando las cosas no salen como yo quiero. & & & & \\
\hline 12 & Ultimamente noto que me cuesta más hacer las tareas y no estudio como antes & & & & \\
\hline 13 & Tengo discusiones con mis padres porque no quieren que este con mis amigos más tiempo. & & & & \\
\hline 14 & Estoy resentido con mis padres porque no me entienden y comprenden. & & & & \\
\hline 15 & Hay compañeros cercanos que se ha ido alejando de mí en los últimos tiempos. & & & & \\
\hline 16 & Me siento alejando de mí mismo, no soy el de antes. & & & & \\
\hline 17 & Estoy desilusionado y triste debido a la actitud de las personas que me rodean. & & & & \\
\hline 18 & Tengo miedo de perder la razón & & & & \\
\hline 19 & Mis compañeros y amigos tienen más suerte que yo en todo & & & & \\
\hline 20 & A pesar de que gustaría llorar, no lo hago para demostrar que soy más fuerte que ellos. & & & & \\
\hline 21 & Me siento feliz de tener una verdadera familia & & & & \\
\hline 22 & $\begin{array}{l}\text { Por las noches reflexiono acerca de mis comportamientos inadecuados para no hacerlos } \\
\text { después. }\end{array}$ & & & & \\
\hline 23 & Sufro demasiado que me gustaría acabar con este sufrimiento de alguna manera. & & & & \\
\hline 24 & Me mortifica que mis amigos pasen más tiempo con otros que conmigo. & & & & \\
\hline 25 & Me siento estresado porque mis padres son muy exigentes conmigo en todo. & & & & \\
\hline 26 & Estoy tan resentido con mis padres que me gustaría no levantarme al día siguiente. & & & & \\
\hline 27 & He perdido el interés en muchas actividades que antes hacía con entusiasmo & & & & \\
\hline 28 & Me siento triste porque mis padres no me expresan su cariño y amor como antes & & & & \\
\hline 29 & Tengo pensamientos de hacerme daño para que mis padres se interesen en mi & & & & \\
\hline
\end{tabular}


30 Hay personas que amo pero no se interesan por mí.

31 Estoy temeroso porque mi rendimiento académico no es el esperado por mis padres

32 Cada vez que voy al colegio me siento ansioso porque me fastidian mucho mis compañeros.

33 Con facilidad me pongo nervioso y no puedo controlarlo

34 Cada vez que me pongo nervioso mis compañeros me fastidian porque se hace notorio mi

34 nerviosismo

35 Mi nerviosismo me juega malas pasadas porque no respondo aun sabiendo la respuesta.

\section{Anexo 3:}

\section{Validez y confiabilidad de la escala de agresividad}

Análisis de items en las dimensiones de la escala de agresividad

\begin{tabular}{lcc}
\hline & \multicolumn{2}{c}{ Escala } \\
\hline Vub - escalas & $\mathbf{R}$ & $\mathbf{P}$ \\
\hline Hostilidad indirecta & $.670^{* *}$ & .001 \\
\hline Irritabilidad & $.521^{* *}$ & .001 \\
\hline Negativismo & $.690^{* *}$ & .001 \\
\hline Resentimiento & $.580^{* *}$ & .001 \\
\hline Recelo & $.683 * *$ & .001 \\
Hostilidad verbal & $.725^{* *}$ & .001 \\
\hline
\end{tabular}

** La correlación es significante al nivel 0,01

Estimaciones de la Confiabilidad de la Escala de agresividad

\begin{tabular}{lcc}
\hline Dimensiones & N $^{\circ}$ de ítems & Alpha \\
\hline Violencia & 10 & 0.6552 \\
\hline Hostilidad indirecta & 9 & 0.6282 \\
\hline Irritabilidad & 11 & 0.6437 \\
\hline Negativismo & 5 & 0.6775 \\
\hline Resentimiento & 8 & 0.6614 \\
\hline Recelo & 10 & 0.6658 \\
Culpalidad verbal & 13 & 0.6487 \\
\hline Escala Total & 9 & 0.6433 \\
\hline
\end{tabular}




\section{Anexo 4:}

\section{Validez y confiabilidad de la escala estado emocional}

\section{Análisis de items en la escala del estado emocional}

\begin{tabular}{|c|c|c|c|}
\hline $\mathbf{N}^{\mathrm{a}}$ & Íttems & $\mathbf{R}$ & Nse \\
\hline 1 & $\begin{array}{l}\text { Reconozco que he sido más agresivo de lo normal con mis compañeros del aula, pero } \\
\text { después me he arrepentido. }\end{array}$ & 0.875 & 0.000 \\
\hline 2 & Tengo pensamientos de que los demás son mejor que yo y eso me pone de mal humor. & 0.821 & 0.000 \\
\hline 3 & $\begin{array}{l}\text { El nivel económico en mi familia no cubre todo lo que a mi me gustaría tener y eso me llena } \\
\text { de ira por que no puedo tener lo que mis compañeros tienen (juegos, propinas, etc.) }\end{array}$ & 0.877 & 0.000 \\
\hline 4 & Me he sentido culpable por haber perjudicado involuntariamente a mis compañeros. & 0.812 & 0.000 \\
\hline 5 & Me suelo emocionar fácilmente. Creo que soy una persona sensible. & 0.910 & 0.000 \\
\hline 6 & Me gusta estar solo (a) y poder relajarme a escondidas de mis padres. & 0.792 & 0.000 \\
\hline 7 & Últimamente me siento solo y salgo menos con los amigos (as) de antes. & 0.789 & 0.000 \\
\hline 8 & Pienso que la vida es bastante aburrida. & 0.798 & 0.000 \\
\hline 9 & Para tomar decisiones necesito consultar a mis amigos(as). & 0.845 & 0.000 \\
\hline 10 & Pienso que dios es injusto por el tipo de vida que llevo. & 0.924 & 0.000 \\
\hline 11 & Soy tolerante cuando las cosas no salen como yo quiero & 0.788 & 0.000 \\
\hline 12 & últimamente noto que me cuesta mas hacer las tareas y no estudio como antes & 0.866 & 0.000 \\
\hline 13 & Tengo discusiones con mis padres porque no quieren que este con mis amigos más tiempo. & 0.834 & 0.000 \\
\hline 14 & Estoy resentido con mis padres porque no me entienden y comprenden. & 0.801 & 0.000 \\
\hline 15 & Hay compañeros cercanos que se ha ido alejando de mí en los últimos tiempos. & 0.844 & 0.000 \\
\hline 16 & Me siento alejando de mí mismo, no soy el de antes. & 0.755 & 0.000 \\
\hline 17 & Estoy desilusionado y triste debido a la actitud de las personas que me rc & 0.830 & 0.000 \\
\hline 18 & Tengo miedo de perder la razón & 0.909 & 0.000 \\
\hline 19 & Mis compañeros y amigos tienen más suerte que yo en todo & 0.866 & 0.000 \\
\hline 20 & A pesar de que gustaría llorar, no lo hago para demostrar que soy más fuerte que ellos. & 0.871 & 0.000 \\
\hline 21 & Me siento feliz de tener una verdadera familia & 0.739 & 0.000 \\
\hline 22 & $\begin{array}{l}\text { Por las noches reflexiono acerca de mis comportamientos inadecuados para no hacerlos } \\
\text { después. }\end{array}$ & 0.819 & 0.000 \\
\hline 23 & Sufro demasiado que me gustaría acabar con este sufrimiento de alguna manera. & 0.916 & 0.000 \\
\hline 24 & Me mortifica que mis amigos pasen más tiempo con otros que conmigo. & 0.856 & 0.000 \\
\hline 25 & Me siento estresado porque mis padres son muy exigentes conmigo en todo. & 0.888 & 0.000 \\
\hline 26 & Estoy tan resentido con mis padres que me gustaría no levantarme al día siguiente. & 0.766 & 0.000 \\
\hline 27 & He perdido el interés en muchas actividades que antes hacía con entusiasmo & 0.925 & 0.000 \\
\hline 28 & Me siento triste porque mis padres no me expresan su cariño y amor como antes & 0.777 & 0.000 \\
\hline 29 & Tengo pensamientos de hacerme daño para que mis padres se interesen en mi & 0.815 & 0.000 \\
\hline 30 & Hay personas que amo pero no se interesan por mí. & 0.787 & 0.000 \\
\hline 31 & Estoy temeroso porque mi rendimiento académico no es el esperado por mis padres & 0.841 & 0.000 \\
\hline 32 & Cada vez que voy al colegio me siento ansioso porque me fastidian mucho mis compai & 0.760 & 0.000 \\
\hline 33 & Con facilidad me pongo nervioso y no puedo controlarlo & 0.870 & 0.000 \\
\hline 34 & $\begin{array}{l}\text { Cada vez que me pongo nervioso mis compañeros me fastidian porque se hace notorio mi } \\
\text { nerviosismo }\end{array}$ & 0.822 & 0.000 \\
\hline 35 & Mi nerviosismo me juega malas pasadas porque no respondo aun sabiendo la respuesta. & 0.787 & 0.000 \\
\hline
\end{tabular}




\begin{tabular}{lcc}
\hline & $\mathbf{N}^{\circ}$ de ítems & Alpha \\
\hline Escala Total & 35 & 0.7236 \\
\hline
\end{tabular}

\section{REFERENCIAS BIBLIOGRÁFICAS}

Ávila A. R. (1998). Estadística elemental. Lima-Perú: Estudios y Ediciones S.A.

Araujo, E. (2013). Cuestionario de personalidad situacional: estudio psicométrico y comparativo en adolescentes de lima. Universidad César Vallejo. Rev. Psicol. Vol.16 No2. Extraído el 08 de agosto del 2016 desde http:/ucvvirtual.edu.pe/contenido_web/ Docs_Adic/20141103_REVPSICO2014-II.pdf

Barrio V, D; Moreno R, C; y López M, R (2001). Evaluación de la agresión y la inestabilidad emocional en niños españoles: su relación con la depresión Clínica y Salud, vol. 12, núm. 1, pp. 33-50.

Bettencourt, B. A., \& Miller, N. (1996). Gender differences in aggressionas a function of provocation: A meta-analysis. Psychological Bulletin, 119, 422- 447.

Buss A. H., y Durkee A. (1987). An inventory for assessing different kinds of hostility. J Consult Psychiatry 21: 343-8.

Buss, A. H., \& Perry, M. P. (1992). The aggression questionnaire. Journal of Personality and Social Psychology, 63, 452-459.

Cerezo, A. (2007). La agresividad: factores determinantes. España: Omega

Chóliz, M. (2005). Psicología de la Emoción: el proceso emocional (pp. 1-33). España. Departamento de Psicología Básica. Universidad de Valencia. Extraído el 13 Junio, 2016, de www.uv.es/=choliz.

Contini, E. N., Imach, S. C., Coronel C, P. y Mejail, S. (2012). Agresividad y retraimiento en adolescentes. Ciencias Psicológicas 2012; VI (1): 17 - 28.

Garaigordobil, M. (2005). Conducta antisocial durante la adolescencia: Correlatos socio emocionales, predictores y diferencias de género. Psicología Conductual, 13(2), 197215 .

Gutiérrez, C., Hernández, V., Sebastián , J y Suárez , A. (2000). Relación entre rasgos de personalidad y conducta antisocial en función de variables sociodemográficas de un grupo de barristas de fútbol. Cuadernos hispanoamericanos de psicología, Vol 12 $N^{o} 1,37-52$.

Hernández, R . (2010). Metodología de la investigación. México:Mc. Graw-Hill.

Kerlinger, N. (2004). Enfoque conceptual de la investigación del comportamiento. Nueva Editorial Interamericana. México.

Majluf, A. (1999). Psicopatología en adolescentes de Lima según el Inventario de Problemas Conductuales de Achenbach. Revista de Psicología de la PUCP, 17(1), 47-71. 
Martínez P; Morote, R. (2001). Preocupaciones de adolescentes de Lima y sus estilos de afrontamiento Pontificia Universidad Católica del Perú Revista de Psicología de la PUCP. Vol. XIX, 2.

Matalinares M; Arenas C; Sotelo L; Díaz G; Dioses A., Yaringaño J; Muratta R; Pareja C; Tipacti R. (2010). Clima familiar y agresividad en estudiantes de secundaria de lima metropolitana. Revista IIPSI UNMSM. Vol. 13 - N. ${ }^{\circ} 1$.

Mestre, V; Samper, P; Frias, D. (2004). Personalidad y contexto familiar como factores predictores de la disposición prosocial y antisocial de adolescentes. Rev. latinoam. Psicol;36(3):445-457, dic.

Olweus, D. (2004). Conductas de acoso y amenaza entre escolares. Ediciones Morata, S.L Madrid. España. pags. 24-25.

Parra, R. (1998). La Escuela Violenta. Bogotá: Tercer Mundo.

Pérez, M. Á., Redondo, M., \& León, L. (2008). Aproximaciones a la emoción de la ira: de la conceptualización a la intervención psicológica. Revista electrónica de motivación y emoción, XI (28).

Peñaherrera, E. (1998). Comportamiento de riesgo adolescente: Una aproximación psicosocial. Revista de Psicología de la PUCP, 16(2), 265-293.Quiroz, N.; Villatoro, J. A.; Juárez, F.; Gutiérrez, $M$.

Reyes, C. (1978). Cuestionario Modificado de Agresividad de BUSS-DURKEE. Universidad Ricardo Palma. Editado ACP. Extraído el 11 Febrero, 2016, de https:// www.monografias.com/docs/Cuestionario-Modificado-De-Agresividad-De-BUSSF3YNWNFZMY

Vila, A., Díaz, L. (2007). Factores de riesgo intrafamiliar, personalidad, conducta emocional y dependencia al internet en estudiantes de una universidad privada. Tesis de maestría en psicología Universidad de San Martin de Porres. Perú: USMP 
